\title{
Interfacing Complexitor: An Empirical-based Educational Tool for Learning Time Complexity
}

\author{
Oscar Karnalim ${ }^{\# 1}$, Elvina ${ }^{\# 2}$ \\ Faculty of Information Technology \\ Maranatha Christian University \\ Bandung, Indonesia \\ loscar.karnalimeit.maranatha.edu \\ ${ }^{2}$ elvinazhangegmail.com
}

\begin{abstract}
Since learning algorithm time complexity in theoretical manner is rather difficult, an educational tool, which is named Complexitor, tried to incorporate empirical approach for teaching such material. Students can learn how to determine a time complexity for given algorithm based on the actual execution. Students are only required to provide algorithm implementation and input set. This paper extends the work of Complexitor by providing a stable interface and qualitative evaluation. The interface is developed based on input and output characteristic of Complexitor whereas the evaluation is represented as a survey toward 20 undergraduate students. Based on student's perspective, Complexitor features, at some extent, may help students to learn algorithm time complexity. Moreover, they also state that our tool has fulfilled standard application aspects. In other words, our tool is eligible to be used for learning algorithm time complexity.
\end{abstract}

Keywords-software; educational tool; time complexity; empirical approach; qualitative evaluation

\section{INTRODUCTION}

According to the fact that student capabilities of learning course material vary, several teaching methods were proposed to handle that issue. One of such methods is providing an educational tool. Using such tool, students are expected to learn given course material based on their capabilities. They can set the teaching speed based on to their learning pace. In addition, they can also repeat difficult parts as many as they like, without worrying that such behavior may annoy other students.

In Computer Science (CS) major, educational tool is also integrated to help students understand course material. Yet, most of them are focused to algorithm topic since such topic is the core subject required for learning other CS courses. In term of encouraging students to understand given material, several approaches are incorporated on CS-based educational tools. Some of them are Program Visualization [1, 2, 3], Visual Programming [4, 5], and Algorithm Visualization [6, 7].

Among numerous CS-based educational tools, there is a tool which is focused on teaching algorithm complexity in empirical manner [8]. That tool, which is named Complexitor, is expected to help students understand how to calculate time complexity based on the real execution. However, the recent work about Complexitor was only focused on a prototype application and technical evaluation. Therefore, this paper extends their work by providing a stable interface and evaluating such tool based on student's perspective. The proposed interface is synchronized with input and output of Complexitor whereas evaluation is conducted based on questionnaire survey toward 20 undergraduate students.

\section{RELATED WORKS}

As Computer Science (CS) students, learning algorithm is mandatory since most CS courses incorporate algorithm as the major requirement for learning their material. However, due to various degree of student capabilities to understand such topic, in-class lecture session may be not sufficient enough to fulfill student needs. Several students require more time to understand than others whereas some of them require more detailed explanation than others. Therefore, to overcome these impediments, several algorithm-centric CS educational tools were developed. These tools target various aspects in learning algorithm. It varies from algorithm implementation to algorithmic steps.

When learning algorithm implementation such as source code, most educational tools were focused on Program Visualization (PV). PV is expected to help students understand their own code through animation and visualization $[1,2,3]$. Such tool is typically featured with reversible code tracking so that students can see how given algorithm works and verify involved variables during that process. Several examples of educational tools which fell into this category are PythonTutor [1], Jeliot 3 [3], and Ville [2]. Among these examples, PythonTutor is the only tool which was exclusively developed as a web application.

Based on the fact that the grammar of programming language is quite strict, most students experience difficulties when preparing their algorithm implementation to be fed on PV tools. Some of them cannot create an error-free source code since they are not used to source code syntaxes. As a result, several educational tools with unique features were introduced. Firstly, Radosevic et al [9] proposed Verificator, an educational tool which limits the number of source code modification per compilation phase. Students cannot compile their own code when the number of source code modification is higher than a particular threshold. In other words, such mechanism forces students to compile and check the validity of their code syntaxes frequently. Secondly, Carlisle et al [4] and Watts [5] proposed 
Visual Programming as a replacement of PV in their educational tools. Visual Programming is a mechanism which prevent students for generating syntaxes errors by applying visual flowchart and drag-and-drop system. Students are not encouraged to write their code directly. Instead, they are only required to pick "code chunk" from a list and drag it to their algorithm implementation. Lastly, Areias \& Mendes [10] proposed careful supervising for teaching weak students. They combined flowchart-like representation and problem-specific questions to guide students.

Instead of focusing on technical features, several algorithmimplementation-based educational tools tried to incorporate "fun" as a part of their tools. Alice [11], CeeBot4 [12], and Karel Robot [13] are several tools which fell into this category. They taught algorithm implementation through 3D object visualization. Students can arrange their own algorithm for available objects and see how their composed algorithm works in an interactive manner. Such fashion is expected to alleviate student's stress when composing an algorithm.

When teaching algorithmic steps, several educational tools incorporated Algorithm Visualization (AV) to visualize how algorithm works in more intuitive manner [14]. Such tool is frequently used to teach popular algorithms such as searching and sorting. A prominent example of such tool is VisuAlgo, a web-based AV portal which covers various algorithm and data structure materials $[15,16,6]$. It incorporated HTML5 as its main resource to animate and visualize algorithm steps. Similar to VisuAlgo, AP-ASD1 [7] also incorporated animation and visualization. Nevertheless, their work was designed as an offline application and more focused on covering course materials.

In term of teaching algorithm, several educational tools are not only designed to teach how an algorithm works but also to teach why a particular algorithm is better than others. So far, these were five works which focused on such goal. Firstly, GreedEx, an educational tool for learning Greedy algorithm [17]. Such tool, which was proposed by Velázquez-Iturbide \& Pérez-Carrasco, can be used to explore how several Greedy algorithms work in empirical manner. Students can see and compare the result of various Greedy algorithms toward specific cases. Secondly, GreedExCol [18], which was expanded from GreedEx by incorporating Computer-Supportive Collaborative System (CSCL). This tool enables students to discuss and determine the best Greedy algorithm for handling a particular problem in collaborative manner. Thirdly, AP-SA, an AV tool for learning 4 algorithm strategies: brute force, greedy, backtracking, and dynamic programming [19]. This tool incorporated case-based performance comparison which, at some extent, may help students to compare algorithm characteristic toward given test case. Fourthly, AP-BB, an AV tool for learning branch \& bound strategy [20]. This tool was featured with comparative review between branch $\&$ bound and brute force strategy per case study. Student can learn why branch $\&$ bound strategy is better than brute force based on given review. Lastly, Complexitor, an educational tool for learning time complexity in empirical manner [8]. Students can learn how an algorithm is assigned with a complexity constant based on empirical evidence.
Despite the fact that Complexitor's empirical approach for determining time complexity yields a promising result, its recent work was only focused on a prototype application and technical evaluation of given empirical approach. Thus, this paper proposes a further development of Complexitor. Stable interface of such tool is introduced based on Complexitor's input and output. In addition to that, our work also incorporates qualitative evaluation to measure the impact of Complexitor based on student's perspective.

\section{METHODOLOGY}

In general, learning flowchart of Complexitor can be seen on Figure 1 [8]. Students should provide three inputs which are target source code (i.e. algorithm implementation), input set, and programming language setting. The first two inputs are casedependent whereas the latter one is programming-languagedependent. Based on these inputs, Complexitor will generate two kinds of number sequences which are execution and complexity-defined sequence. Execution sequence is generated based on either the number of processed instruction or execution time through actual execution whereas complexity-defined sequences are generated by executing standard complexity functions toward input size. After both sequences are generated, time complexity for the target algorithm is assigned with complexity which sequence is the most correlated one toward execution sequence. Correlation between sequence is conducted based on Pearson correlation [21].

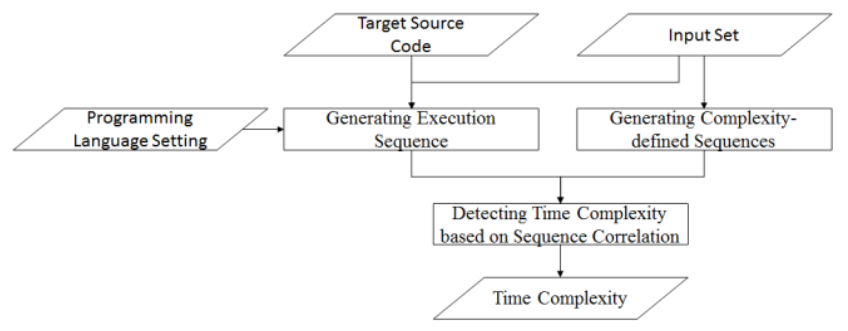

Figure 1. Learning Flowchart of Complexitor [8]

According to given flowchart, the interface of Complexitor is designed as in Figure 2. Its design is adapted from our previous Algorithm Visualization works [19, 7] since, based on our survey, this typical design was successfully engaged students in our major for learning algorithm-related topics. On main window, there are five components which are problem configuration, source code viewer, counter integration, programming language setting, and miscellaneous. All components are represented as A to E on Figure 2 respectively.

Before utilizing Complexitor, students must choose which mechanism is incorporated for detecting time complexity empirically. Student can select either the number of involved instructions (NI-GES) or time execution (TI-GES) on problem configuration. Afterwards, student can load algorithm implementation and its input set on the same component. Loaded algorithm implementation would be shown on source code viewer so that students can see directly its selected algorithm implementation. When students select NI-GES for detecting time complexity, students must select which lines are involved for counter initialization, increment, and print. This task can be conducted either by typing involved line numbers manually on 
counter integration section or by selecting targeted lines using mouse pointer on source code viewer. For convenience, selected lines would be displayed with different color on source code viewer. Line for counter initialization is displayed with blue color; Lines for counter increment are displayed with purple color; and Line for counter print is displayed with green color.



Figure 2. The Interface of Complexitor

It is important to note that selecting targeted lines is conducted manually based on twofold. Firstly, such mechanism may encourage students to understand more about how to measure time complexity in empirical manner. Students should know where the calculation of instruction starts and ends through counter initialization and counter print. Counter increment, on the other hand, may encourage students to understand which instructions are involved for determining time complexity. Secondly, incorporating automatic mechanism to detect involved lines may require students to provide advanced programming syntaxes and grammar, which are impractical, especially for undergraduate students who have limited prior knowledge about programming.

Beside choosing which mechanism to use for measuring time complexity, students should also provide input set where each input case must be represented as a file text. Students can add or modify available input cases on list from left-side component. For convenience, each input case should follow two simple rules: 1) File name should represent the number of input involved; and 2) The content of given file should only contain program input.

It is clear that input set may affect resulted time complexity greatly due to our empirical manner. Our approach determines time complexity based on real execution of a program toward given input set. Thus, when given input set does not represent a particular scenario (i.e. best-case scenario), our approach would not generate the correct time complexity. Input set can be provided either by the students or lecturer as long as all input cases are designed to generate a particular scenario. Based on our previous work [8], 10 input cases with reasonable delta value between inputs should be enough to detect time complexity accurately.

When students want to incorporate algorithm implementation in other programming language, they can set current programming language on programming language setting. In general, there are 5 components which should be defined per programming language. These components are compile instruction, run instruction, counter initialization, counter increment, and counter print. On the one hand, compile and run instruction refer to instructions to compile and run source code through command line. Such instructions can be provided with environment variables for accessing file name and location dynamically. In such manner, students are not required to change programming language setting each time a source code with different file name or location is incorporated. Our Complexitor would detect such features directly and replaces all environment variables with its respective values. In general, three environment variables can be used in programming language setting. These variables are@filepath, @filenamewithextention, and @filenamewithoutextention. The first one refer to absolute path for given source code whereas the other two refer to source code file name. On the other hand, counter initialization, increment, and print are three components which are related to programming language syntaxes. These components are used to detect time complexity through NI-GES.

All components of programming language setting can be stored as a XML file for portability and readability. A sample XML file of $\mathrm{C}++$ Programming language setting can be seen on Figure 3 where each tag represents a component. name tag refers to programming language name; compile tag refers to compile 
instruction; run tag refers to run instruction; dec tag refers to counter initialization; inc tag refers to counter increment; and save tag refers to counter print. It is important to note that several special characters are automatically translated to HTML entity to provide safe-parsed XML file. One example of such phenomena is " $<$ " character on save tag which is translated into "\&lt".

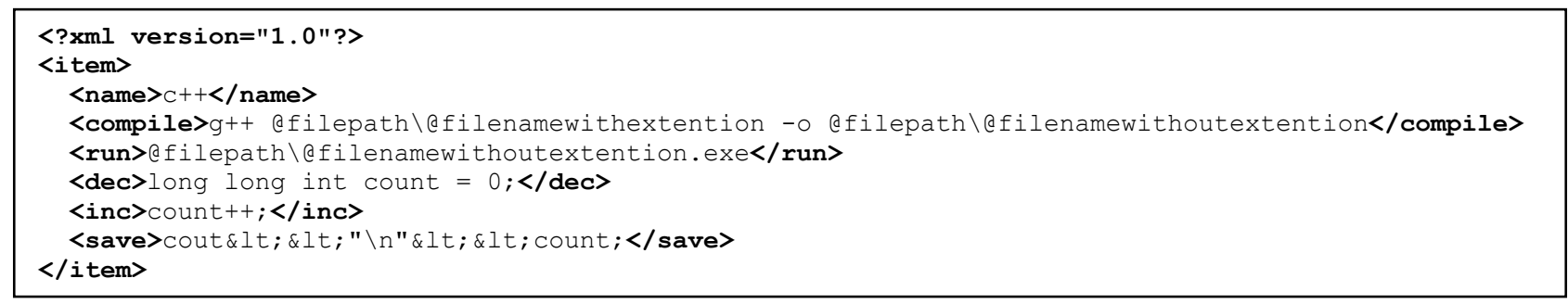

Figure 3. Sample XML File for C++ Programming Language

In order to provide more detail about how our Complexitor works, the sample view of Complexitor when all fields are filled right before its time complexity would be calculated can be seen on Figure 4. It was taken when the complexity of linear search would be measured. As seen in Figure 4, 10 test cases were incorporated with various $\mathrm{N}$. It starts from $\mathrm{N}=1$ to $\mathrm{N}=1000$ and all of them were designed to perform worst-case scenario. Since the linear search was implemented in Java programming language, programming language setting on the right side showed the detail of Java setting. Counter instruction such as counter initialization, increment, and print would be embedded on given source code based on marked lines on source code viewer. In this case, counter initialization would be embedded at the end of line 3; counter increment would be embedded at the end of line 5, 6, 7, 11 and 24; and counter print would be embedded at the end of line 25 . It is important to note that not all instructions must be involved for determining time complexity. Given linear search on Figure 4 only considers linear search method for determining time complexity and ignoring its input mechanism.

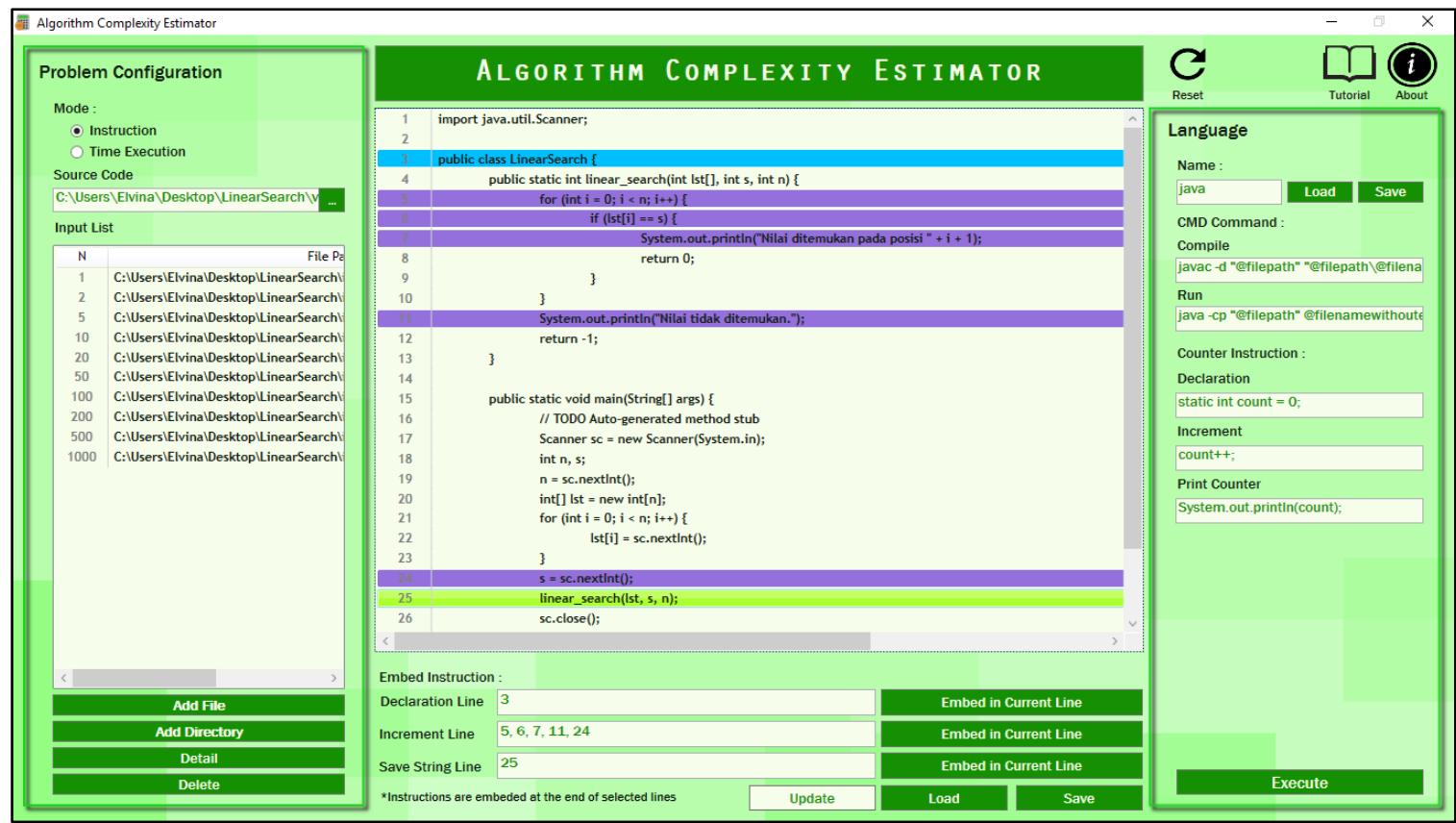

Figure 4. The Sample View of Complexitor with Filled Fields

After algorithm implementation is executed for each given input case, Complexitor would display predicted time complexity in a pop-up window which view can be seen on Figure 5. The predicted time is featured with two supplementary information which are sequence comparison and calculated correlation value. Sequence comparison displays the difference between complexity-defined and execution sequence so that students can see how similar both sequences are. Such information is displayed on the left side as a two-column table.
First column refers to complexity-defined sequence whereas the second one refers to execution sequence. Calculated correlation value, on the contrary, displays Pearson correlation value between execution sequence to all available complexity-defined sequence. It is represented as two-column table which is placed at the right side of pop-up window. Such information is expected to provide sufficient information about why such complexity is defined as the result of given process. 


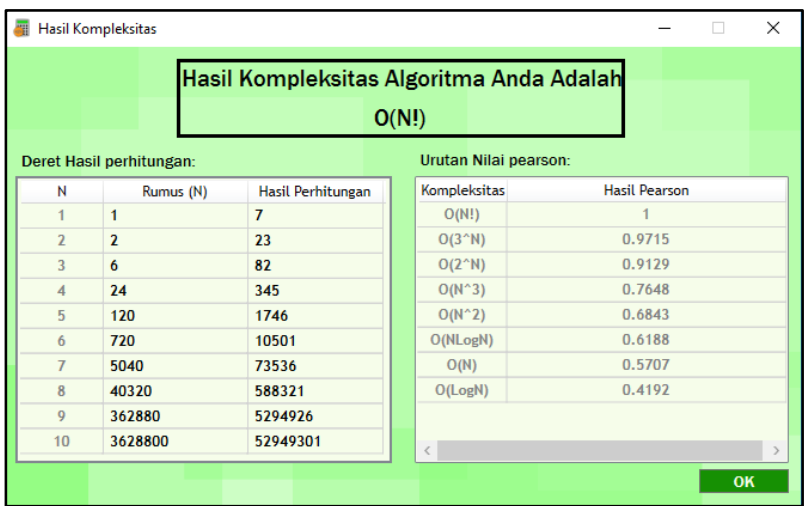

Figure 5. Sample View of Resulted Time Complexity on Complexitor

Miscellaneous component consists of three features which are Reset, Tutorial, and About. Reset is intended to clear all provided fields on Complexitor; Tutorial is intended to provide a comprehensive information about how to learn time complexity with Complexitor; and About is intended to provide developer information and contact. The sample view of Tutorial section can be seen in Figure 6 which summarizes how to use Complexitor for learning time complexity. It starts from preparing all required inputs to theoretical foundation about learning time complexity using algorithm implementation and input set.

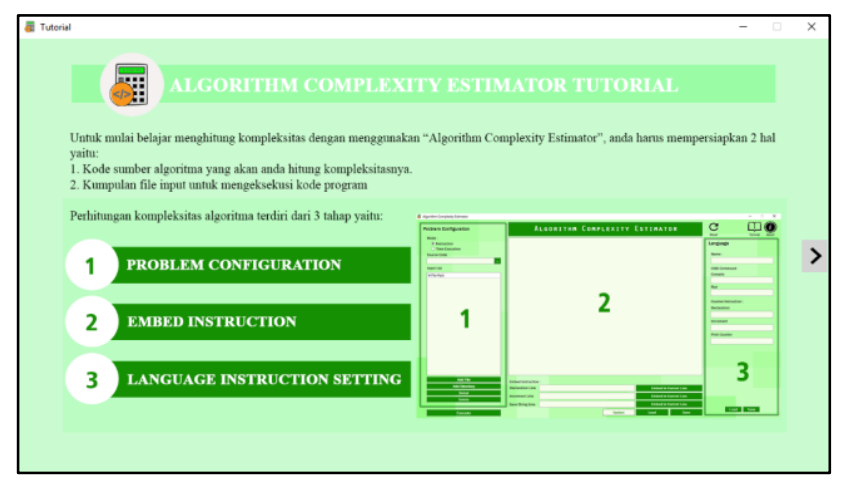

Figure 6. A Sample View of Complexitor Tutorial

\section{Evaluation}

To prove the effectiveness of Complexitor, particularly on user perspective, a survey was conducted to 20 respondents. These respondents are 20 undergraduate students from Faculty of Information Technology, Maranatha Christian University, Indonesia. However, in order to keep the validity of our survey result, we limited our respondents to students who had already taken Algorithmic Strategy course in their previous semesters. Algorithmic Strategy is a CS-based course which incorporates algorithm complexity as one of its syllabus material. In such manner, all respondents should have known about algorithm complexity in general. This prior knowledge is required since Complexitor assumes that students have already known several time complexity terminologies.

Our questionnaire survey consists of 9 statements where each one of them should be answered on 7-points Likert scale (1 represents strongly disagree; 4 represents neutral; and 7 represents strongly agree). The detail of our questionnaire survey statements can be seen on Table I. For convenience, each statement is assigned with unique ID and will be referred as its ID at the rest of this paper. Before answering questionnaire survey, each respondent was given a comprehensive information about Complexitor by the second author of this paper. Such information was given as a presentation that includes live simulation of how Complexitor works.

\section{TABLE I. QUESTIONNAIRE STATEMENTS}

\begin{tabular}{|c|l|}
\hline ID & \multicolumn{1}{|c|}{ Statement } \\
\hline Q1 & $\begin{array}{l}\text { When generating execution sequence with the number of } \\
\text { instructions (NI-GES approach), counter initialization and print } \\
\text { encourage students to learn where to start and end calculating } \\
\text { time complexity }\end{array}$ \\
\hline Q2 & $\begin{array}{l}\text { When generating execution sequence with the number of } \\
\text { instructions (NI-GES approach), counter increment encourages } \\
\text { students to learn which instructions are included for determining } \\
\text { algorithm time complexity }\end{array}$ \\
\hline Q3 & $\begin{array}{l}\text { Determining time complexity in empirical manner (sequence } \\
\text { correlation) is easier to understand than the theoretical one. }\end{array}$ \\
\hline Q4 & $\begin{array}{l}\text { Determining time complexity based on execution time helps } \\
\text { students to understand how to calculate algorithm time } \\
\text { complexity further. }\end{array}$ \\
\hline Q5 & $\begin{array}{l}\text { Determining time complexity based on the number of instructions } \\
\text { helps students to understand how to calculate algorithm time } \\
\text { complexity further. }\end{array}$ \\
\hline Q6 & $\begin{array}{l}\text { Complexitor User Interface fulfills user necessity in terms of } \\
\text { learning algorithm time complexity }\end{array}$ \\
\hline Q7 & $\begin{array}{l}\text { Complexitor functionality fulfills user necessity in terms of } \\
\text { learning algorithm time complexity }\end{array}$ \\
\hline Q8 & Complexitor tutorial is declarative \\
\hline Q9 & $\begin{array}{l}\text { In general, Complexitor may help students to learn algorithm } \\
\text { time complexity }\end{array}$ \\
\hline
\end{tabular}

The mean score of each statement in our survey can be seen on Figure 7. In general, all statements were agreed by the respondents since all statements yielded score greater than 5 (tend to agree). However, among these statements, Q1 yielded the highest mean value which is 5.8 of 7 . In other words, counter mechanism applied in our approach was the most prominent feature for our respondents, especially for determining the start and end state for calculating time complexity. Start state is represented as counter initialization whereas the end state is represented as counter print. They believed that such mechanism might help students for learning time complexity in empirical manner. Following Q1, Q8 and Q9 yielded the second-highest mean score. Both of them resulted 5.75 of 7 , which was slightly lower than Q1 score. The high mean value of both statements yield the fact that our tutorial and general intention of Complexitor were accepted well by the respondents. They believed that such tutorial is declarative and the general use of Complexitor may help students for learning algorithm time complexity. Q5, on the other hand, yielded the lowest mean value which is 5.15 of 7 . When discovered further, most respondents tended to give moderate score due to the lack of execution log toward given algorithm implementation. According to our respondents, such execution log, which records executed lines as a sequence, may help students to understand why an algorithm with selected instructions may provide a particular time complexity. They doubt that providing the result directly is the most effective approach to teach algorithm time 
complexity. Not all students have acknowledged the very-basic concept of algorithm time complexity, particularly involved instructions for determining given time complexity. However, since Q5 mean score is still higher than 5, it can be stated that our approach for determining time complexity based on involved instruction still has a positive impact for students.

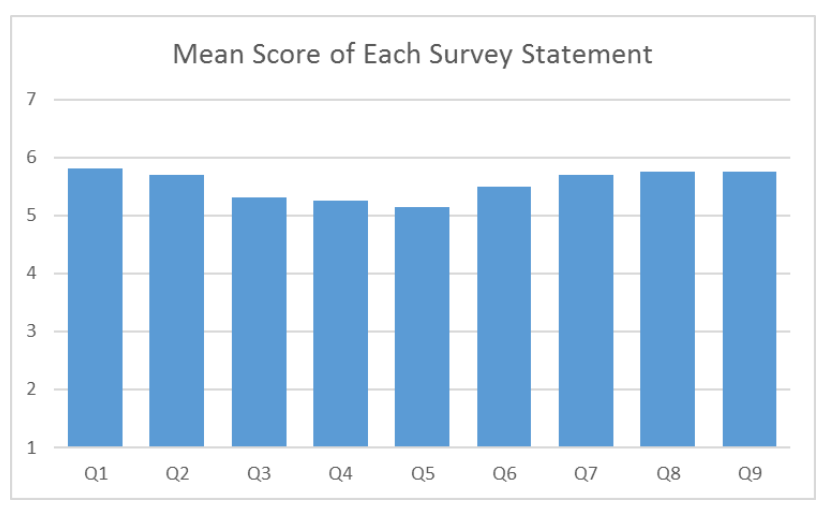

Figure 7. Mean Score of Each Survey Statement

In order to measure the variation between respondent's answers, our work also measured the standard deviation of each statement in our survey. The detail of resulted standard deviation can be seen on Figure 8. On the one hand, Q1 yielded the lowest standard deviation among all statements. Thus, it can be stated that our respondents tended to have the most similar response of Q1. On the other hand, Q4 yielded the highest standard deviation, which indirectly stated that the response of respondents might be varied. When discovered further, there were two contradicting views about involving real execution time for learning time complexity. Some of them believed that execution time is more practical than the number of instructions since students are not required to set involved instructions. Whereas, the others believed that execution time may not sufficient to help students due to its inaccuracy. However, since the mean score of Q4 is still higher than 5, it can be concluded that time execution was still agreed as the prospective mechanism to help students learning time complexity, even though it does not provide a significant impact.

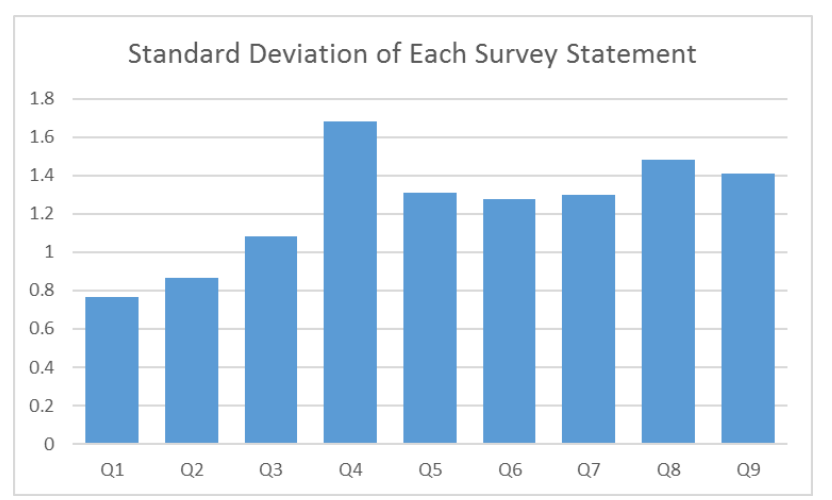

Figure 8. Standard Deviation of Each Survey Statement

Q1 and Q2 were incorporated to evaluate the impact of counter initialization, increment, and print for learning to calculate time complexity based on the number of instructions. Based on Table I, both Q1 and Q2 yielded high mean and low standard deviation. Thus, it can be concluded that most respondents tend to agree with Q1 and Q2. Incorporating counter mechanism semi-manually may help students to learn time complexity.

Q3, Q4, and Q5 were incorporated to strengthen our assumption that determining time complexity in empirical manner (sequence correlation) is easier to understand than the theoretical one. Q3 strengthened our assumption in general whereas Q4 and Q5 strengthened our assumption on either time execution or the number of instructions for learning time complexity. According to our survey result, all of them yielded high mean (score $\geq 5$ ) which conclude that sequence correlation approach is easier to understand than the theoretical one. Q4 yielded higher score than Q5 since most respondents thought that real time execution is easier to understand than the number of involved instruction. It uses similar terminology with the concept of algorithm time complexity. However, since real time execution may generate inaccurate result [8], they also believed that the number of instructions should be involved as a replacement of real time execution.

Q6 to Q9 were incorporated to evaluate standard application aspects on Complexitor. Q6 was intended to evaluate User Interface; Q7 was intended to evaluate functionality; Q8 was intended to evaluate tutorial section; and Q9 was intended to evaluate overall impact. Similar with other statements, all statements in this category were agreed by respondents, which means that our Complexitor has passed standard application aspects. The standard deviation of these statements was high since the definition of application aspects are rather subjective and different for each respondent.

\section{CONCLUSION AND FUTURE WORK}

In this paper, we have interfaced Complexitor, an educational tool for learning algorithm time complexity in empirical manner. Students can learn how to calculate time complexity based on actual execution of algorithm implementation. Based on our qualitative evaluation, Complexitor matches our expectation for fulfilling student necessities since all evaluated statements yield mean score higher than 5 (tend to agree). In general, we can conclude three findings which were agreed by the respondents. Firstly, counter mechanism on NI-GES approach from Complexitor is beneficial for learning time complexity. It was concluded from Q1 and Q2 survey statements. Secondly, empirical approach is more understandable than the theoretical one. It was concluded from Q3 to Q5 survey statements. Finally, Complexitor has already fulfilled user necessity for learning algorithm time complexity in general. It was concluded from Q6 to Q9 survey statements.

For further research, we will conduct a controlled experiment for evaluating the impact of Complexitor on Algorithmic Strategy course in our university. In addition, we also intend to extend Complexitor with theoretical learning approach so that students can learn how to calculate time complexity from both empirical and theoretical manner.

\section{REFERENCES}

[1] P. J. Guo, "Online python tutor: embeddable web-based program visualization for cs education," in The 44th ACM technical symposium on Computer science education, Denver, 2013. 
[2] T. Rajala, M.-J. Laakso, E. Kaila and T. Salakoski, "Effectiveness of Program Visualization : A case study with the ViLLE Tool," Journal of Information Technology Education : Innovation in Practice, vol. 7, 2008.

[3] S. M. Cisar, R. Pinter, D. Radosav and P. Cisar, "Software visualization: The educational tool to enhance student learning," in The 33rd International Convention MIPRO, 2010.

[4] M. C. Carlisle, T. A. Wilson, J. W. Humphries and S. M. Hadfield, "RAPTOR: a visual programming environment for teaching algorithmic problem solving," in The 36th SIGCSE technical symposium on Computer science education, St. Louis, 2005.

[5] T. Watts, "The SFC editor a graphical tool for algorithm development," Journal of Computing Sciences in Colleges, vol. 20, no. 2, 2004.

[6] S. Halim, Z. C. Koh, V. B. H. Loh and F. Halim, "Learning Algorithms with Unified and Interactive Web-Based Visualization," Olympiads in Informatics, vol. 6, pp. 53-68, 2012.

[7] L. Christiawan and O. Karnalim, "AP-ASD1: An Indonesian Desktopbased Educational Tool for Basic Data Structures," Jurnal Teknik Informatika dan Sistem Informasi (JuTISI), vol. 2, no. 1, 2016.

[8] E. Elvina and O. Karnalim, "Complexitor: An Educational Tool for Learning Algorithm Time Complexity in Practical Manner," ComTech: Computer, Mathematics and Engineering Applications, vol. 8, no. 1, 2017.

[9] D. Radosevic, T. Orehovacki and A. Lovrencic, "Verificator: Educational Tool for Learning Programming," Informatics in Education, vol. 8, no. 2, 2009.

[10] C. Areias and A. Mendes, "A tool to help students to develop programming skills," in The 2007 international conference on Computer systems and technologies, Bulgaria, 2007.

[11] S. Cooper, W. Dann and R. Pausch, "Alice: a 3-D tool for introductory programming concepts," Journal of Computing in Small Colleges, vol. 15 , no. 5,2000

[12] "Learn programming with CeeBot4," 59 2008. [Online]. Available: http://www.ceebot.com/ceebot/4/4-e.php. [Accessed 511 2016].
[13] D. Buck and D. J. Stucki, "JKarelRobot: a case study in supporting levels of cognitive development in the computer science curriculum," in The thirty-second SIGCSE technical symposium on Computer Science Education, Charlotte, 2001.

[14] C. A. Shaffer, M. L. Cooper, A. J. D. Alon, M. Akbar, M. Stewart, S. Ponce and S. H. Edwards, "Algorithm Visualization: The State of the Field," ACM Transactions on Computing Education (TOCE), vol. 10, no. 3, 2010.

[15] S. Halim, "VisuAlgo," [Online]. Available: http://visualgo.net/. [Accessed 125 2015]

[16] E. T. Y. Ling, Teaching Algorithms with Web-based Technologies, Singapore: B.Comp. Dissertation, Department of Computer Science, School of Computing, National University of Singapore, 2014.

[17] J. Á. Velázquez-Iturbide and A. Pérez-Carrasco, "Active learning of greedy algorithms by means of interactive experimentation," in ITiCSE '09 Proceedings of the 14th annual ACM SIGCSE conference on Innovation and technology in computer science education, New York, 2009.

[18] O. Debdi, M. Paredes-Velasco and J. Á. Velázquez-Iturbide, "GreedExCol, A CSCL tool for experimenting with greedy algorithms," Computer Applications in Engineering Education, vol. 23, no. 5, pp. 790804, 2015.

[19] F. C. Jonathan, O. Karnalim and M. Ayub, "Extending The Effectiveness of Algorithm Visualization with Performance Comparison through Evaluation-integrated Development," in Seminar Nasional Aplikasi Teknologi Informasi, Yogyakarta, 2016.

[20] S. Zumaytis and O. Karnalim, "Introducing An Educational Tool for Learning Branch \& Bound Strategy," Journal of Information Systems Engineering and Business Intelligence, vol. 3, no. 1, 2017.

[21] K. Pearson, "Notes on regression and inheritance in the case of two parents," in The Royal Society of London, 1895. 